\title{
Effects of Mo Addition on the Mechanical Properties and Microstructures of Ti-Mn Alloys Fabricated by Metal Injection Molding for Biomedical Applications
}

\author{
Pedro Fernandes Santos ${ }^{1}$, Mitsuo Niinomi ${ }^{2,3,4,5, *}$, Ken Cho ${ }^{3}$, Huihong Liu ${ }^{6}$, Masaaki Nakai ${ }^{7}$, \\ Takayuki Narushima ${ }^{1}$, Kyosuke Ueda ${ }^{1}$ and Yoshinori Itoh $^{8}$ \\ ${ }^{1}$ Graduate School of Engineering, Tohoku University, Sendai 980-8579, Japan \\ ${ }^{2}$ Graduate School of Science and Technology, Meijo University, Nagoya 468-8502, Japan \\ ${ }^{3}$ Graduate School of Engineering, Osaka University, Suita 565-0871, Japan \\ ${ }^{4}$ Institute of Materials Research, Tohoku University, Sendai 980-8577, Japan \\ ${ }^{5}$ Materials and Systems for Sustainability, Nagoya University, Nagoya 464-8603, Japan \\ ${ }^{6}$ Joining and Welding Research Institute, Osaka University, Ibaraki 567-0047, Japan \\ ${ }^{7}$ Department of Mechanical Engineering, Faculty of Science and Engineering, Kindai University, Higashiosaka 577-8502, Japan \\ ${ }^{8}$ Hamamatsu Technical Support Center Industrial Research Institute of Shizuoka Prefecture, Hamamatsu 431-2103, Japan
}

Ti-Mn alloys fabricated by metal injection molding (MIM) show promising performance for biomedical applications, but their low ductility (caused by high $\mathrm{O}$ content and the presence of pores and carbides) requires improvement. Previously, the addition of Mo to cold crucible levitation melted (CCLM) Ti-Mn alloys efficiently improved the ductility of those alloys by promoting mechanical twinning. In the present study, Mo was added to Ti-Mn alloys fabricated by MIM. Unlike fabrication by CCLM, fabrication by MIM can produce alloys with a smaller grain size, and also introduce microstructures such as pores and Ti carbides. Thus, in order to investigate how Mo addition interacts with these typical MIM features, four alloys for biomedical applications were fabricated by MIM: Ti-5Mn-3Mo (TMM-53), Ti-5Mn-4Mo (TMM-54), Ti6Mn-3Mo (TMM-63), and Ti-6Mn-4Mo (TMM-64). Their microstructures, mechanical properties, and tensile deformation mechanisms were evaluated. Their hardness values range from 312-359 HV, and their Young's modulus values range from 84-88 GPa; both the Vickers hardness and Young's modulus show little variation among the alloys. Although the alloys show fracture features associated with a predominantly ductile fracture mode and Mo addition successfully promotes mechanical twinning in TMM-54, the elongation of these alloys is still critically low. Compared to the TMM alloys fabricated by CCLM, the TMM alloys fabricated by MIM show slightly lower hardness and Young's modulus, and comparable tensile strength, with their low elongation remaining inadequate for such applications. In particular, TMM-63 shows the best combination of mechanical properties among the present alloys, with an elongation of $4 \%$ and an ultimate tensile strength of $1145 \mathrm{MPa}$. [doi:10.2320/matertrans.M2016286]

(Received August 18, 2016; Accepted November 21, 2016; Published January 16, 2017)

Keywords: titanium-manganese-molybdenum alloys, $\beta$-phase, mechanical properties, deformation mechanisms, metal injection molding

\section{Introduction}

Ti and its alloys have a more suitable biocompatibility and combination of mechanical properties than other metallic materials used as implant biomaterials, such as SUS316L steel and Co-Cr-Mo alloys ${ }^{1)}$. Although high specific strength (strength-to-density ratio), high corrosion resistance, and high biocompatibility make Ti and its alloys attractive materials for biomedical applications ${ }^{2,3}$, some Ti alloys can release metallic ions that are harmful to the human body. Among the most used Ti-based biomaterials are commercially-pure Ti (CP-Ti) and Ti-6Al-4V (Ti-64) ELI ${ }^{1)}$. However, these materials have significant issues specific to their application as structural biomaterials. CP-Ti has relatively low mechanical strength compared to other metallic biomaterials such as Co-Cr-Mo alloys ${ }^{4)}$. On the other hand, Ti-64 ELI, which is used because of its good mechanical properties, releases $\mathrm{V}$ and $\mathrm{Al}$ ions in biological media ${ }^{5)}$ which are toxic ${ }^{6-8)}$. Another problem observed in metallic biomaterials used in hard tissue fixation or replacement is the mismatch between the Young's modulus of the metallic implant and that of the cortical human bone. The Young's modulus of Ti-64 ELI, although lower than those of steels and Co-Cr-Mo alloys ${ }^{1)}$, is $110 \mathrm{GPa}$; that of cortical human bone is only $10-30 \mathrm{GPa}^{9}$.

*Corresponding author, E-mail: niinomi@imr.tohoku.ac.jp, Emeritus Professor, Tohoku University
Such a mismatch can cause stress shielding effects ${ }^{10)}$, which prevent loads that are essential for the health of the bone tissue, leading to bone resorption and a decrease in the quality of the bone around the implant $t^{3,11)}$.

In recent years, increased efforts have been directed at producing more biocompatible $\mathrm{Ti}$ alloys ${ }^{2)}$. These alloys are mainly $\beta$-type alloys composed of non-toxic elements, with a Young's modulus of $50-90 \mathrm{GPa}^{4,12-14)}$. The $\beta$-phase in Ti exhibits a lower Young's modulus than the other phases, while having a highest specific strength ${ }^{15)}$. One recently developed alloy, Ti-29Nb-13Ta-4.6Zr (TNTZ), shows excellent mechanical properties and biocompatibility for biomedical applications ${ }^{2,9,16)}$. Unfortunately, both $\mathrm{Nb}$ and Ta are elements regarded as critical materials, meaning that these alloying elements have important applications in various industrial fields and as such have scarcity issues because of their limited amount of natural deposits around the globe ${ }^{17,18)}$.

In previous studies, Mn was chosen as an alloying element because of its $\beta$-stabilizing effect, lower cytotoxicity, and higher availability compared to other alloying elements ${ }^{19-21)}$. Low-cost Ti-Mn alloys were fabricated using a metal injection molding (MIM) process $^{21}$, a near-net shape advanced powder metallurgy $(\mathrm{P} / \mathrm{M})$ fabrication method which reduces the amount of waste material to by as much as $1 / 5$ compared to conventional melting-machining methods ${ }^{22)}$, reducing fabrication costs. Ti-9Mn (mass\%) showed the best balance of mechanical properties among both the alloys fabricated by 
cold crucible levitation melting (CCLM $)^{19)}$ and those fabricated by $\mathrm{MIM}^{21)}$. Nevertheless, the ductility of the alloys warrants improvement ${ }^{19,21)}$.

In another recent study, Mo was added as an alloying element to Ti-Mn alloys fabricated by CCLM in order to improve their ductility and the balance of their mechanical properties $^{23)}$. Ti-Mn-Mo (TMM) alloys show improved balance in their mechanical properties, and some alloys which show significant $\{332\}<113>$ mechanical twinning showed significant improvement in their ductility by twinning-induced plasticity (TWIP) ${ }^{23)}$. Some other merits of Mo addition to Ti-Mn systems, including improved corrosion properties, were also discussed ${ }^{23)}$.

Compared to Ti-Mn alloys fabricated by CCLM $^{19)}$, fabrication by MIM introduces pores, carbides, and high interstitial impurity contents, which cause a drastic reduction in the tensile ductility of the Ti-Mn alloys ${ }^{21)}$. Conversely, Mo addition improves the tensile ductility of alloys fabricated by CCLM by promoting TWIP under tensile deformation. Therefore, investigations must be conducted to clarify whether the ductility of the alloys fabricated by MIM can also be improved by TWIP through Mo addition. In this study, Mo was included as an alloying element in Ti-Mn alloys fabricated by MIM in order to evaluate its effects. Chemical compositions similar to those of alloys fabricated by CCLM were used in this study, i.e., Ti-(5-6)Mn-(3-4)Mo (mass\%). The microstructure, Vickers hardness, Young's modulus, and tensile properties of the alloys were investigated along with their deformation mechanisms.

\section{Experimental Procedures}

\subsection{Material preparation}

Sintered specimens of Ti-(5-6)Mn-(3-4)Mo were fabricated by MIM. Hereafter, the Ti-Mn-Mo alloys will be labeled as "TMM" followed by their nominal alloying contents, e.g., Ti-5Mn-3Mo is TMM-53 and Ti-6Mn-4Mo is TMM-64. The alloys were prepared using gas-atomized $\mathrm{Ti}$ and fine Mn powders with particles sizes $<45 \mu \mathrm{m}$, and fine Mo powder with particles sizes $<2 \mu \mathrm{m}$. Figure 1 shows typical scanning electron microscopy (SEM) images of the Ti, Mn, and Mo powders, having O impurity levels of $0.16,0.77$, and 0.38 mass $\%$ respectively. Additionally, due to the possibility of Mn evaporation during sintering process ${ }^{21)}$, additional small quantities of Mn were added to the raw mixed powders. The powders were mixed with an organic binder and injection molded into rectangular specimens measuring approximately $80 \mathrm{~mm}$ long, $10 \mathrm{~mm}$ wide, and $4 \mathrm{~mm}$ thick. The binder was partially removed with $n$-hexane and then thermal debinding was performed; this injection molding process has been described previously ${ }^{24)}$. The sintering was conducted in a vacuum furnace at $1373 \mathrm{~K}$ for $28.8 \mathrm{ks}$. The alloys were then subjected to solution treatment in a vacuum at $1173 \mathrm{~K}$ for $3.6 \mathrm{ks}$ followed by ice water quenching. The solutionized alloys were subsequently cut into smaller specimens for microstructural observation, analysis and mechanical characterization.

\subsection{Microstructural characterization}

The metallic compositions of the TMM alloys were analyzed by inductively coupled plasma optical emission spec- (a)

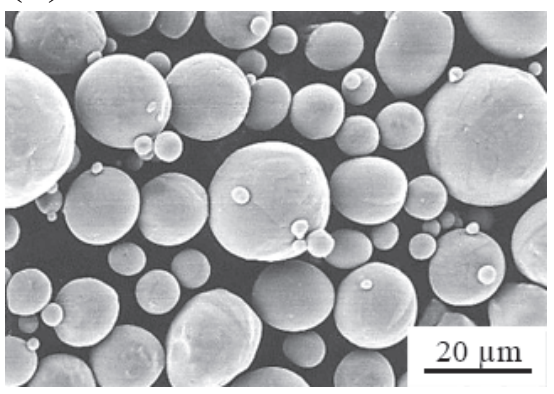

(b)

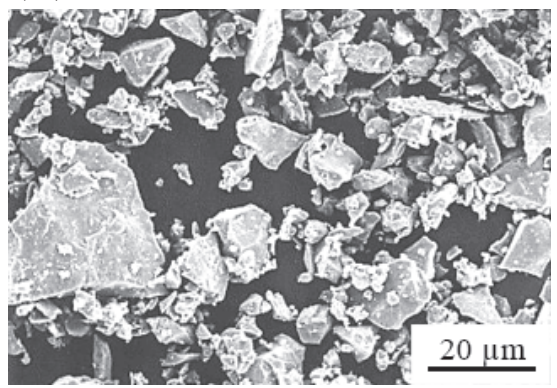

(c)

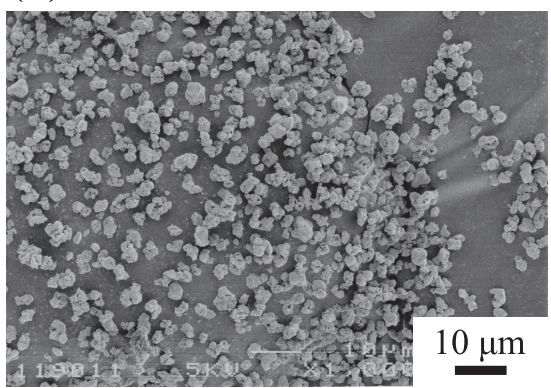

Fig. 1 SEM images of (a) Ti, (b) Mn, and (c) Mo powders used for fabricating TMM alloys.

troscopy (ICP-OES). Infrared (IR) absorption was used to determine the $\mathrm{O}$ and $\mathrm{C}$ compositions. The microstructures of the TMM alloys were investigated using an optical microscope (OM). OM specimens were mechanically polished using waterproof $\mathrm{SiC}$ papers up to \#2400 grit and mirror-polished using a colloidal silica suspension, then etched using a $\left(1 \% \mathrm{HF}+0.5 \% \mathrm{HNO}_{3}\right)$ solution. The average grain diameters, $D_{g r}$, were estimated from optical micrographs using an image analysis software.

The constituent phases of the TMM alloys were investigated by X-ray diffraction (XRD) and transmission electron microscopy (TEM). XRD specimens were mechanically polished using waterproof $\mathrm{SiC}$ papers up to \#2400 grit, and then mirror-polished using a colloidal silica suspension. XRD analyses were performed using a $\mathrm{Cu}$ target, an acceleration voltage of $40 \mathrm{kV}$, and a current of $40 \mathrm{~mA}$. TEM observations were conducted on thin circular foils $3 \mathrm{~mm}$ in diameter. These thin foils were mechanically polished to a thickness of approximately $30 \mu \mathrm{m}$ using a \#2400 grit waterproof $\mathrm{SiC}$ paper and then dimpled to a thickness of approximately $10 \mu \mathrm{m}$ using a phosphor bronze ring, followed by ion milling the films. TEM observations were carried out with an acceleration voltage of $200 \mathrm{kV}$. 


\subsection{Mechanical tests}

The Vickers hardness, $H v$, values of the TMM alloys were measured using a micro-Vickers hardness testing machine with a load of $9.807 \mathrm{~N}$ and dwell time of $15 \mathrm{~s}$. The specimens used for the hardness measurements were mechanically polished using waterproof $\mathrm{SiC}$ papers with grits of up to $\# 2400$, and mirror-polished using a colloidal silica suspension.

The Young's modulus, E, measurements were carried out using a free resonance method. The specimens used for the Young's modulus measurements were $60.0 \mathrm{~mm}$ long, $7.5 \mathrm{~mm}$ wide, and $1.8 \mathrm{~mm}$ thick, and were mechanically polished using waterproof $\mathrm{SiC}$ papers with grits of up to \#1500.

The tensile properties were measured using an Instron-type testing machine with a cross-head speed of $8.33 \times 10^{-6} \mathrm{~m} / \mathrm{s}$. The tensile test specimens were cut using an electrical discharge machining system. The specimens were cut to a gauge length of $10.8 \mathrm{~mm}$, gauge width of $3.5 \mathrm{~mm}$, thickness of $1.8 \mathrm{~mm}$, and fillet radius of $8.0 \mathrm{~mm}$. The specimens were mechanically polished using waterproof $\mathrm{SiC}$ papers with grits of up to \#1500. Strain gauges were used to determine the $0.2 \%$ proof stress. The fracture surfaces of the tensile-tested specimens were observed by SEM. Following the tensile tests, the surfaces of the specimens were examined by electron backscatter diffraction (EBSD) and TEM in order to analyze the deformation products. At least three samples of each alloy were used for each of the above-mentioned tests.

\section{Results}

\subsection{Microstructure}

Table 1 lists the chemical compositions of the TMM alloys, along with the Mo equivalent $\left(M o_{e q}\right)$ calculated as $M o_{e q}=$ $1.7\left[\mathrm{Mn}\right.$ mass \% ] + 1[Mo mass\%]. The concept of $M o_{e q}$ can be used as a measurement of the relative $\beta$-phase stability for the alloys $^{25)}$. Part of the excess Mn did not evaporate during the sintering process, causing the Mn content to be higher than the nominal. The $\mathrm{O}$ contents change according to the $\mathrm{Mn}$ or Mo contents, but this variation is negligible $(0.22-$ 0.24 mass \%). The $\mathrm{C}$ contents of the alloys are approximately 0.08 mass $\%$ for all alloys.

Figure 2 shows optical micrographs of the TMM alloys. The average grain diameters, were estimated from the optical micrographs using image analysis software. There is no significant difference in the $D_{g r}$ of the TMM alloys, which is approximately $37.0 \pm 2.5 \mu \mathrm{m}$. Small closed pores, large interconnected pores, and precipitates are present in each alloy. Both the pores (ellipses) and precipitates (arrows) are mostly located at the grain boundaries.

Figure 3 shows the volume fractions and average diameters

Table 1 Chemical compositions of TMM alloys fabricated by MIM (mass\%), along with calculated $M o_{e q}$.

\begin{tabular}{ccccccc}
\hline \multirow{2}{*}{ Alloy } & \multicolumn{5}{c}{ Element } \\
\cline { 2 - 6 } & Ti & Mn & Mo & O & C & \\
\hline TMM-53 & bal. & 5.20 & 2.95 & 0.222 & 0.077 & 11.79 \\
TMM-54 & bal. & 5.15 & 3.99 & 0.235 & 0.078 & 12.75 \\
TMM-63 & bal. & 6.19 & 3.02 & 0.228 & 0.081 & 13.54 \\
TMM-64 & bal. & 6.18 & 3.96 & 0.244 & 0.074 & 14.47 \\
\hline
\end{tabular}

of the pores, $V_{p o}$ and $D_{p o}$, respectively, and precipitates, $V_{p r}$ and $D_{p r}$, respectively, in the TMM alloys, as estimated from the optical micrographs using an image analysis software. Figure 3(a) shows the $V_{p o}$ and $D_{p o}$, which do not significantly vary among the alloys. The $V_{p o}$ varies between approximately $4.8 \%$ (for TMM-64) and 6.4\% (for TMM-53), while the $D_{p o}$ varies between $10.3 \mu \mathrm{m}$ (TMM-64) and $11.7 \mu \mathrm{m}$ (TMM-53). Figure 3(b) shows the $V_{p r}$ and $D_{p r}$, which also do not signifi-

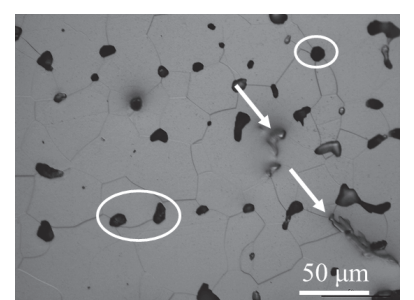

(a)

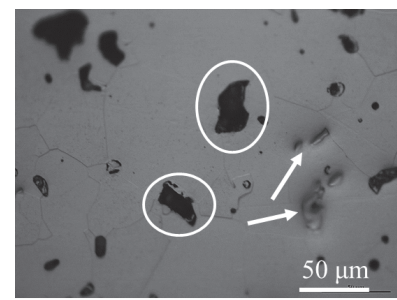

(c)

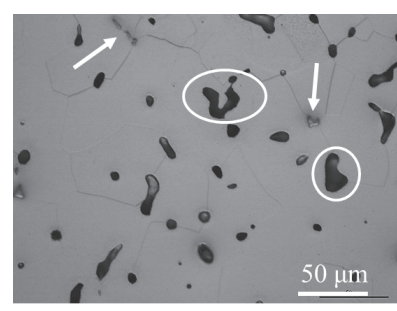

(b)

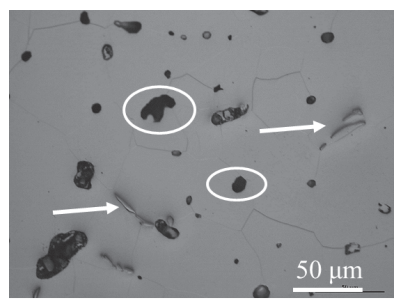

(d)
Fig. 2 Typical optical micrographs of (a) TMM-53, (b) TMM-54, (c) TMM-63, and (d) TMM-64. Some of the pores are indicated by ellipses and some of the precipitates by arrows.

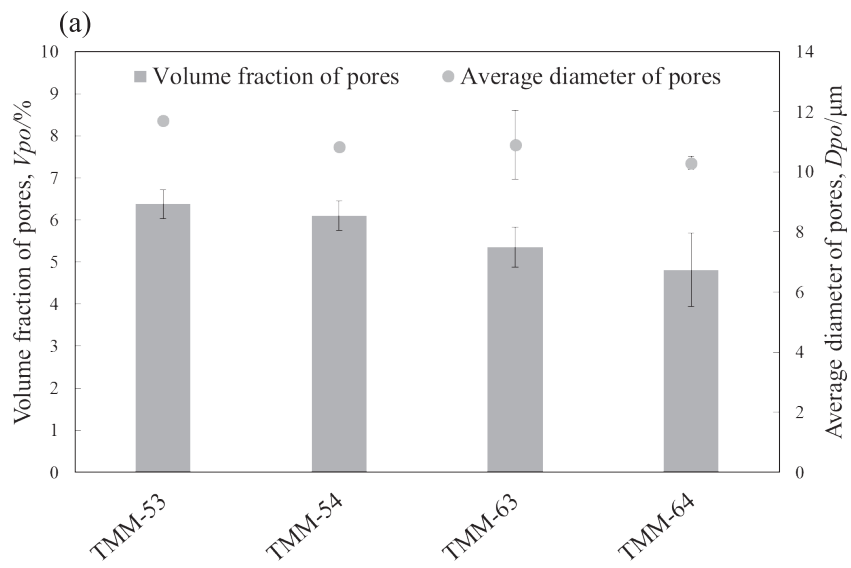

(b)

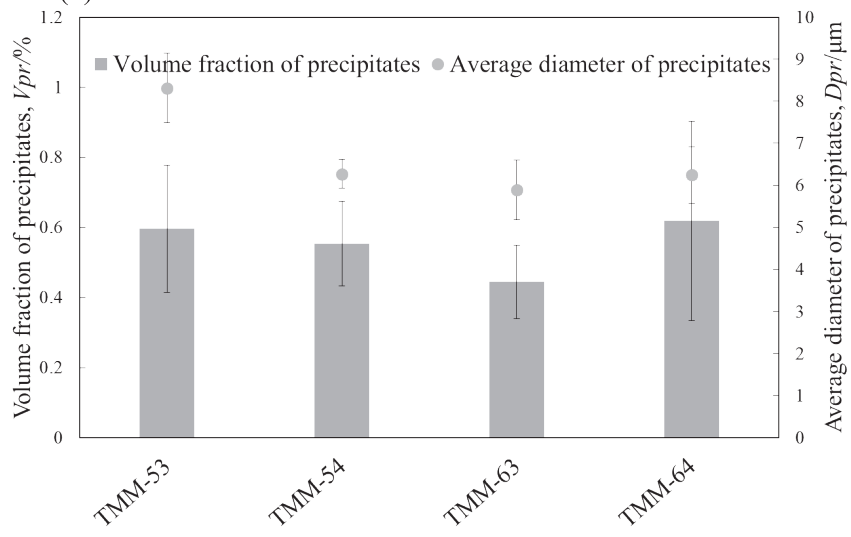

Fig. 3 Volume fractions and average diameters of (a) pores and (b) precipitates in TMM alloys. 
cantly vary among the alloys. The $V_{p r}$ varies between approximately $0.45 \%$ (TMM-63) and $0.62 \%$ (TMM-64), while the $D_{p r}$ varies between $5.9 \mu \mathrm{m}$ (for TMM-63) and $8.3 \mu \mathrm{m}$ (for TMM-53).

Figure 4 shows the XRD profiles of the TMM alloys. Only the diffraction peaks of the $\beta(110), \beta(200), \beta(211)$, and $\beta(220)$ planes are visible in the XRD profiles, indicating that the matrix of the alloys is composed of equiaxed $\beta$-grains.

Figure 5 shows the selected area electron diffraction (SAED) patterns and dark field (DF) images of the diffraction spots or streaks of the $\omega$ phase of the TMM alloys. Diffraction spots produced by the athermal $\omega$ phase can be observed in the SAED patterns of TMM-53 and TMM-54. The $\omega$ diffraction spots become more diffuse with higher Mn contents. Only diffuse streaks associated with the athermal $\omega$ phase can be observed in the SAED patterns of TMM-63 and TMM-64. The DF images of the $\omega$ spots (Fig. 5(e) and (f)) evidence a higher volume fraction of the athermal $\omega$ phase in both TMM53 and TMM-54 than in TMM-63 and TMM-64, based on the small bright particles observed here. The athermal $\omega$ phase particles are more dispersed in the DF images of TMM-63 and TMM-64.

\subsection{Mechanical properties}

Figure 6 shows the Vickers hardness of the TMM alloys, along with that of wrought Ti-64 ELI ${ }^{4}$ indicated by the dashed line. The $H v$ shows negligible variation from TMM-

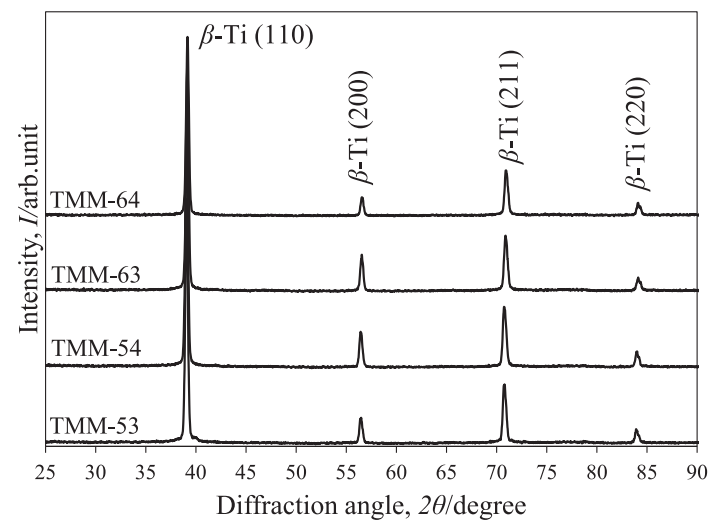

Fig. 4 XRD profiles of TMM alloys.

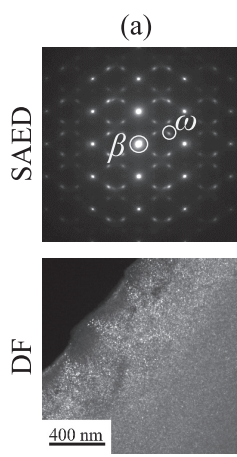

(e) (b)
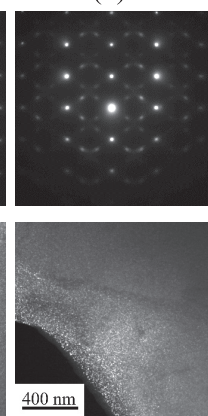

(f) (c)
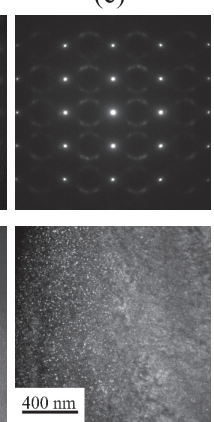

(g) (d)
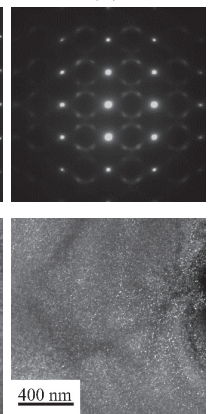

(h)
Fig. 5 Typical SAED patterns viewed from $[110]_{\beta}$, and corresponding DF images of diffraction spots or streaks of $\omega$ phase of: (a) and (e) TMM-53, (b) and (f) TMM-54, (c) and (g) TMM-63, (d) and (h) TMM-64.
53 (331 HV) to TMM-54 (337 HV), increases in TMM-63 (359 HV), and then decreases to its lowest value in TMM-64 (312 HV). The TMM alloys show superior or comparable $H v$ to that of wrought Ti-64 ELI (325 HV).

Figure 7 shows the Young's moduli, E, of the TMM alloys, along with that of wrought Ti-64 ELI ${ }^{4)}$ indicated by the dashed line. The differences among the Young's moduli of the alloys are negligible: TMM-53 (88 GPa), TMM-54 (84 GPa), TMM-63 (86 GPa), and TMM-64 (85 GPa). The Young's moduli of the alloys are lower than that of wrought Ti-64 ELI (110 GPa).

Figure 8 shows the tensile properties of the TMM alloys along with those of wrought Ti-64 ELI ${ }^{4)}$. The ultimate tensile strength (UTS, $\left.\sigma_{B}\right)$ and $0.2 \%$ proof stress $\left(\sigma_{0.2}\right)$ gradually increase from TMM-53 $\left(\sigma_{B}=1036 \mathrm{MPa}, \sigma_{0.2}=980 \mathrm{MPa}\right.$, elongation $=2.0 \%)$ to TMM-54 $\left(\sigma_{B}=1104 \mathrm{MPa}, \sigma_{0.2}=1046 \mathrm{MPa}\right.$, elongation $=1.9 \%)$, and further to TMM-63 $\left(\sigma_{B}=1143\right.$, $\sigma_{0.2}=1077 \mathrm{MPa}$, elongation $\left.=3.9 \%\right)$; these then decrease in TMM-64 $\left(\sigma_{B}=1093 \mathrm{MPa}, \sigma_{0.2}=1033 \mathrm{MPa}\right.$, elongation = $5.0 \%$ ). The elongation increases with increasing $M o_{e q}$ and is highest for TMM-64. The alloys show good tensile strength, higher than that of wrought Ti-64 ELI. However, their elongation is lower than that of wrought Ti-64 ELI. Furthermore, the difference between the $\sigma_{B}$ and $\sigma_{0.2}$ of every alloy is relatively constant (approximately $60 \mathrm{MPa}$ ).

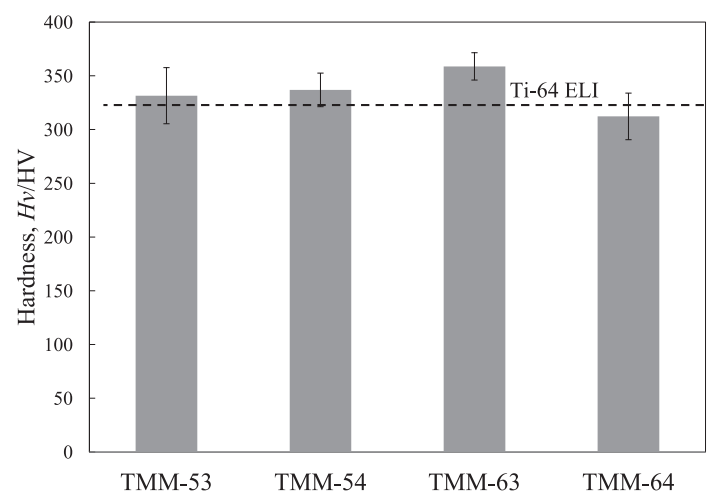

Fig. 6 Comparison of Vickers hardness of TMM alloys with that of wrought Ti-64 ELI (dashed line).

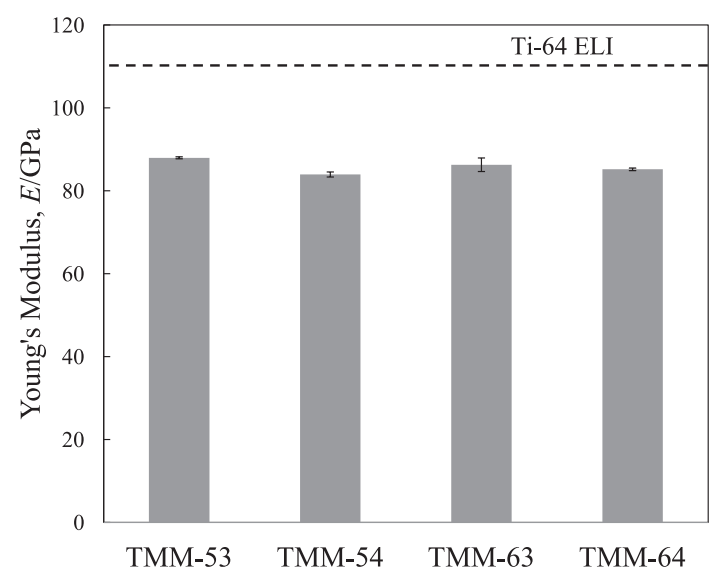

Fig. 7 Comparison of Young's moduli of TMM alloys with that of wrought Ti-64 ELI (dashed line). 


\subsection{Microstructure of tensile-tested specimen}

Figure 9 shows the SEM fractographs of the tensile-tested TMM. There is no significant difference in the fracture surface morphologies or features among the different alloys.

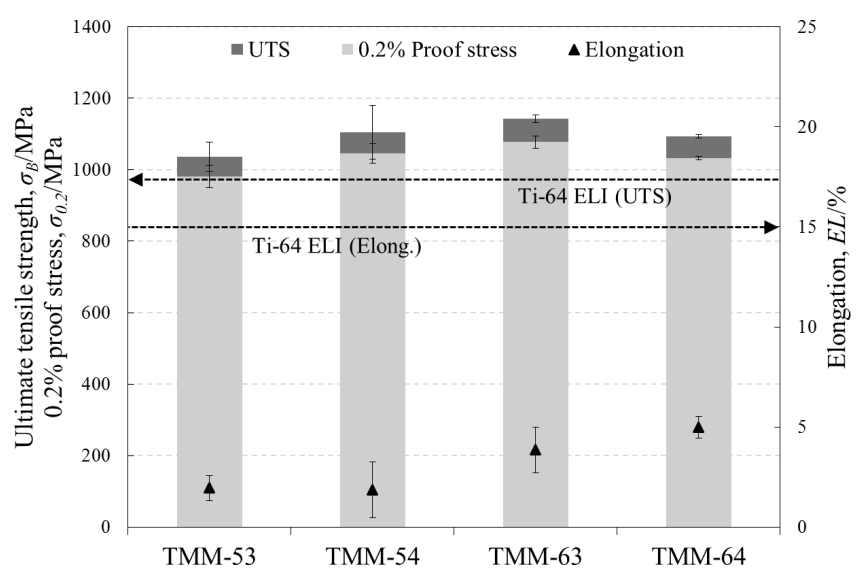

Fig. 8 Comparison of tensile properties of TMM alloys. The properties of wrought Ti-64 ELI are also shown (dashed lines).

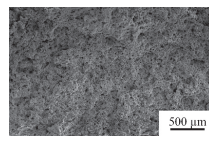

(a)

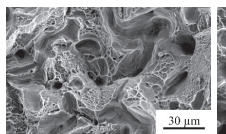

(e)

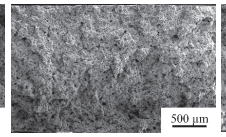

(b)

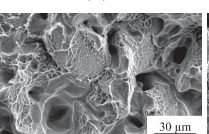

(f)

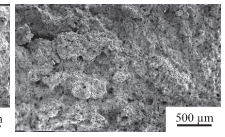

(c)

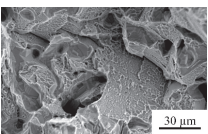

(g)

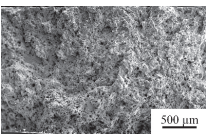

(d)

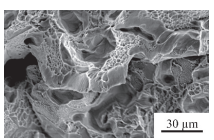

(h)
Fig. 9 Typical SEM fractographs of tensile-tested specimens of: (a) and (e) TMM-53, (b) and (f) TMM-54, (c) and (g) TMM-63, and (d) and (h) TMM-64. Images (e) to (h) are taken at a higher magnification. The fracture surfaces of the alloys are predominantly covered by dimples and large pores.
Pores are clearly visible in all alloys, as well as areas covered in dimples, which are associated with a ductile type of fracture.

Figure 10 shows EBSD inverse pole figure (IPF) maps of the tensile-tested TMM alloys, with the misorientation profile along the arrow connecting points A-B (Fig. 10(b)). For TMM-54 (Fig. 10(b)), a small amount of band structures showing misorientation angles of approximately $50^{\circ}$ are present. These band structures are thus identified as $\{332\}<113>$ mechanical twins ${ }^{26)}$. TMM-53 (Fig. 10(a)), TMM-63 (Fig. 10(c)), and TMM-64 (Fig. 10(d)) show no band-like structures.

Figure 11 shows the SAED patterns and DF images of the diffraction spots of the tensile-tested TMM-53 and TMM-54, along with the key diagrams. Figure 11(a) shows the SAED pattern of the interface between the matrix (with faint $\omega$ phase reflections) and a martensite structure, along with their key diagram. The band structure identified in the DF image of the marked $\alpha$ " spot in the SAED pattern are thus identified as $\alpha$ " martensite ${ }^{27)}$. Figure 11(b) shows the SAED pattern of the interface between the matrix and a band structure which contains both the $\alpha$ " martensite and the twin, along with their key diagram. The $\omega$ phase reflections of the twin can be observed as faint spots, one of which is marked as $\omega_{1}$. The spot marked as $\omega_{m}$, which is the $\omega$ phase reflection from the matrix, overlaps with one $\alpha$ " martensite. Therefore, the center DF image, from the $\omega_{m}$ reflection at a lower magnification level, includes $\alpha$ " martensite particles. The $\alpha$ " martensite appears as the brighter areas inside the band structure of this DF image. The bottom DF image, from the $\omega_{m}$ reflection at higher magnification levels, shows the distribution of $\omega$ particles around the matrix, which is mostly uniform. The right-most DF image, from the $\omega_{1}$ variant reflection, shows the distribution of $\omega$ phases around the twin. This twin shows a misorientation angle of approximately $50^{\circ}$ with the matrix around the $<110>_{\beta}$

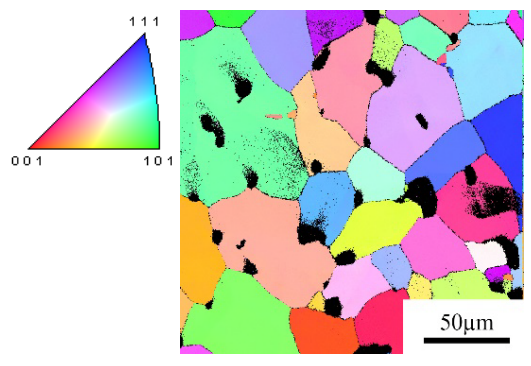

(a)

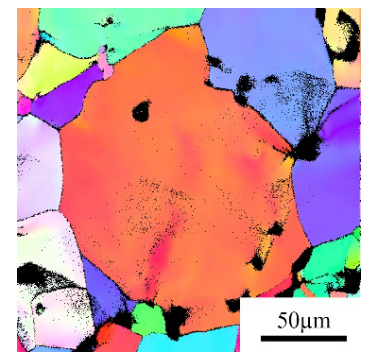

(c)

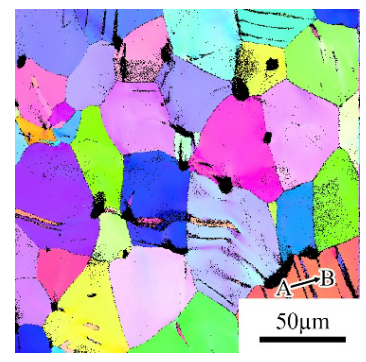

(b)

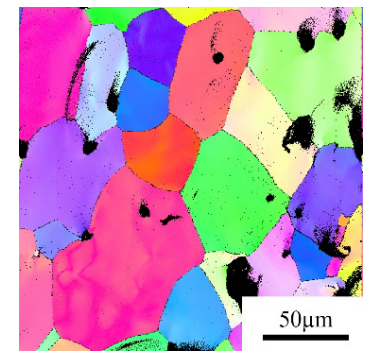

(d)

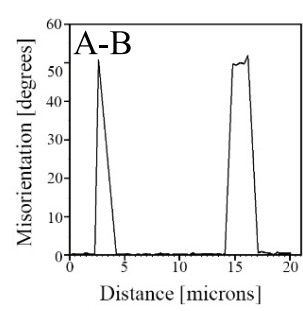

Distance [microns]

Fig. 10 Typical EBSD maps of tensile-tested specimens: (a) IPF map of TMM-53, (b) IPF map and misorientation profile along the arrow between points A-B in TMM-54, (c) IPF map of TMM-63, and (d) IPF map of TMM-64 


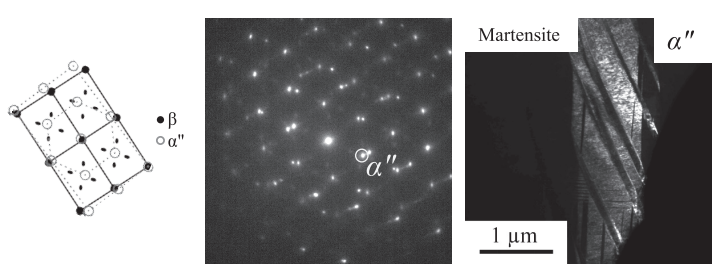

(a)
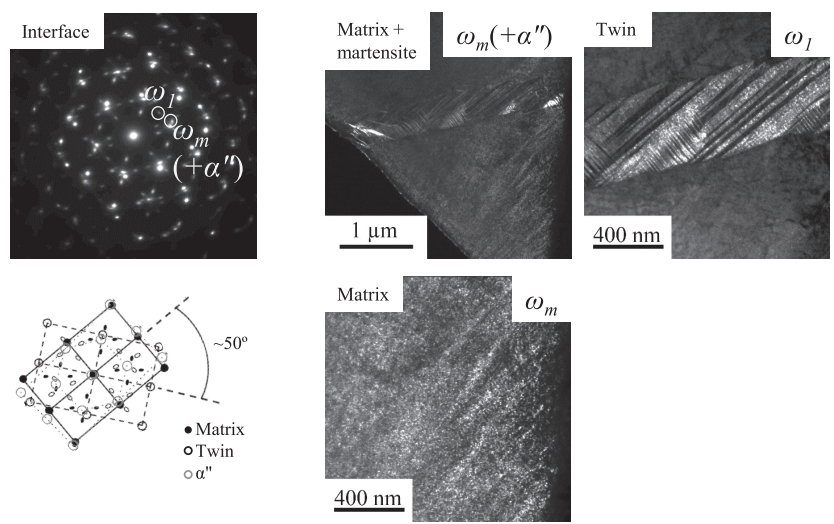

(b)

Fig. 11 TEM observations of tensile-tested specimens: (a) SAED pattern viewed from $[110]_{\beta}$, and key diagram of a matrix-martensite interface of TMM-53, with the DF images of an $\alpha$ " spot observed in the SAED pattern, and (b) SAED pattern viewed from $[110]_{\beta}$, and key diagram of a matrix-band structure (which includes both twin and martensite) interface of TMM-54, with DF images from overlapping $\omega_{m}$ and $\alpha$ " spots, an $\omega_{1}$ spot of the twin, and the $\omega_{m}$ from the matrix.
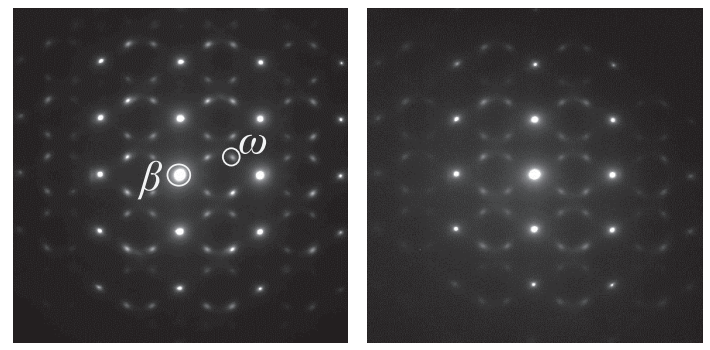

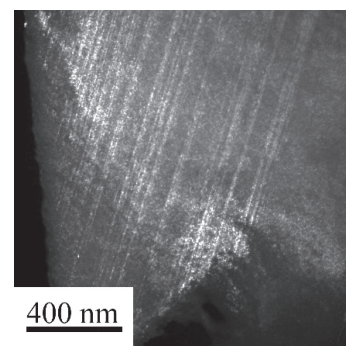

(a)

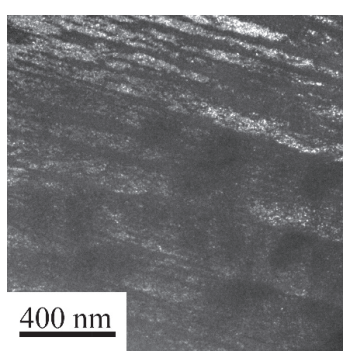

(b)
Fig. 12 TEM observations of tensile-tested specimens: (a) SAED patterns and DF images of $\omega$ spots of TMM-63, and (b) SAED patterns and DF images of $\omega$ spots of TMM-64. The deformation-induced $\omega$ phase lamellae can be observed in both alloys.

direction (indicated by the key diagram), and is identified as a $\{332\}<113>$ mechanical twin.

Figure 12 shows the SAED patterns and DF images of the diffraction spots or streaks of the $\omega$ phase of the tensile-tested TMM-63 and TMM-64 (Fig. 12(a) and (b), respectively). No band-like structures are observed for these alloys. However, groups of thin parallel lamellae, identified as $\omega$ phases, can be observed in both alloys. These deformation-induced $\omega$ lamellae, along with small amounts of $\omega$ nanoparticles in the matrix which were not as clearly observed before tensile deformation (Fig. 5), indicate the presence of a deformation-induced $\omega$ phase in these alloys after tensile deformation.

\section{Discussion}

\subsection{Microstructure}

Most of the $\mathrm{O}$ present in the TMM alloys originates from the $\mathrm{Ti}, \mathrm{Mn}$, and Mo powders, which contain approximately $0.16,0.77$ and 0.38 mass\% O, respectively. However, because the variation in $\mathrm{Mn}$ and $\mathrm{Mo}$ is small, the variation in the $\mathrm{O}$ contents among the TMM alloys is negligible.

The $D_{g r}$ of the TMM alloys fabricated by MIM is much smaller than that of the TMM alloys fabricated by CCLM ${ }^{23}$, and also smaller than that of Ti-Mn alloys fabricated by $\mathrm{MIM}^{21)}$. A number of factors contribute to this smaller grain size observed in TMM alloys fabricated by MIM. The presence of pores in the alloys inhibit grain growth ${ }^{28)}$, but this effect is similar among all the alloys because of the small variation in porosity. The reason for the drastic decrease in $D_{g r}$ compared to that of Ti-Mn alloys fabricated by MIM (which also contains pores) is the presence of Mo. Mo has a low diffusion coefficient in $\mathrm{Ti}^{29)}$, which effectively limits the grain boundary diffusion and growth. In fabrication by MIM, diffusion processes are predominant ${ }^{30)}$. Thus, the low diffusion coefficient of Mo can inhibit grain growth in the TMM alloys fabricated by MIM.

The $V_{p o}$ of the TMM alloys fabricated by MIM show less variation than those observed for Ti-Mn alloys fabricated by $\mathrm{MIM}^{21)}$. Furthermore, the $D_{p o}$ of the TMM alloys is also smaller than that of the Ti-Mn alloys. This is because the Mo powder $(<2 \mu \mathrm{m})$ is smaller than the $\mathrm{Ti}$ and $\mathrm{Mn}$ powders $(<45 \mu \mathrm{m})$. Therefore, Mo powder particles can occupy spaces that would have been left vacant between Ti and Mn powder particles, improving the compactibility and relative density of the mixed powders upon injection molding. Thus, the smaller particle size of the Mo powder can make these pores smaller and more rounded ${ }^{22,31,32)}$. Although the smaller Mo powder had an effect over the pore morphology, no significant direct effect of the Mo element addition over the pore morphology is observed.

Figure 13 shows the BF and SAED patterns of precipitates found in the TMM alloys fabricated by MIM. The SAED patterns indicate that the precipitates possess a typical fcc crystal structure, the same as the Ti-carbides identified in Ti-Mn alloys fabricated by $\mathrm{MIM}^{21)}$. Therefore, the precipitates in the TMM alloys are identified as Ti-carbides, where formation is facilitated by the high levels of $\mathrm{C}$. The morphology of the carbides does not particularly vary among the TMM alloys, and is also similar to that of the Ti-Mn alloys ${ }^{21)}$, indicating negligible effect of the Mo addition in this regard. Figure 14 shows simulations, made using the software ThermoCalc, for the solubility of $\mathrm{C}$ in some of the TMM and Ti-Mn alloys. Because both TMM-64 and Ti-9Mn have a higher $M o_{e q}$ and more stable $\beta$ phase than TMM-53 and Ti-6Mn, respectively, it is apparent that the solubility of $\mathrm{C}$ is more dependent on the 


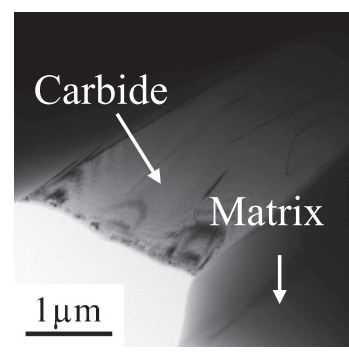

(a)

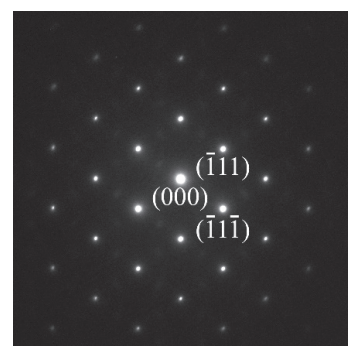

(c)

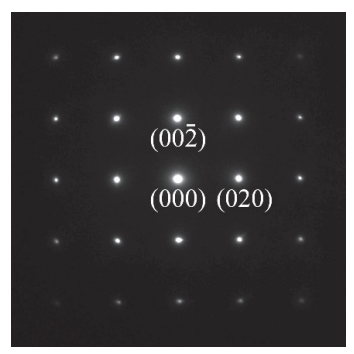

(b)

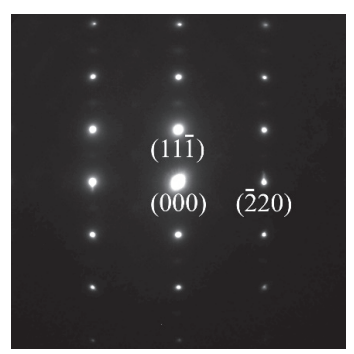

(d)
Fig. 13 Typical (a) BF, and SAED patterns of a Ti carbide viewed from: (b) [100], (c) [110], and (d) [112].

stability of the $\beta$ phase than on which stabilizer element is included. Similar observations were reported for Ti-Nb alloys ${ }^{41)}$

The OM and XRD analyses only show the presence of $\beta$-phase, whereas the presence of an athermal $\omega$ phase is confirmed by TEM observations. As the Mn content increases, the athermal $\omega$-phase reflections decrease in intensity, becoming diffuse streaks in the more $\beta$-stable alloys (Fig. 5(a), (b), (c), and (d) $)^{33,34)}$, indicating that the amount of athermal $\omega$ phase decreases with increasing Mn content. Furthermore, the intensity of the $\omega$ phase reflections appear weaker in the SAED patterns of the TMM alloys fabricated by MIM than in the SAED patterns of the TMM alloys fabricated by CCLM ${ }^{23)}$. This is because higher $\mathrm{O}$ contents will inhibit the formation of the $\omega$ phase $^{35}$. Thus, weaker $\omega$ phase reflections in the SAED patterns of the TMM alloys fabricated by MIM containing approximately 0.24 mass $\% \mathrm{O}$ is also attributed to the higher $\mathrm{O}$ contents.

\subsection{Mechanical properties}

Factors that influence the Vickers hardness of the TMM alloys include the presence of the athermal $\omega$ phase $^{34)}$, pores, and carbides ${ }^{31,32)}$, as well as the solid solution hardening effects of $\mathrm{Mn}, \mathrm{Mo}, \mathrm{O}$ and $\mathrm{C}^{32,36,37)}$. The decreasing volume fraction of the $\omega$ phase tends to decrease the $H v$. Conversely, higher Mn and Mo contents increase the solid solution hardening effect, increasing the $H v$. However, the effect of Mo is negligible compared to that of Mn or the amount of athermal $\omega$ phase $^{36)}$. Thus, as the amount of athermal $\omega$ phase does not significantly change between TMM-53 and TMM-54, the $H v$ is almost the same for those alloys. Then, because increasing Mn, increases the $H v$ in TMM-63 because of the solid solution hardening effect. The reason for the decrease in $H v$ in TMM-64 is unclear, but because of the higher $M o_{e q}$, this alloy is expected to contain a lower amount of the athermal $\omega$ phase, although this is not immediately clear in the DF images. Generally, pores can cause localized stress concentration,
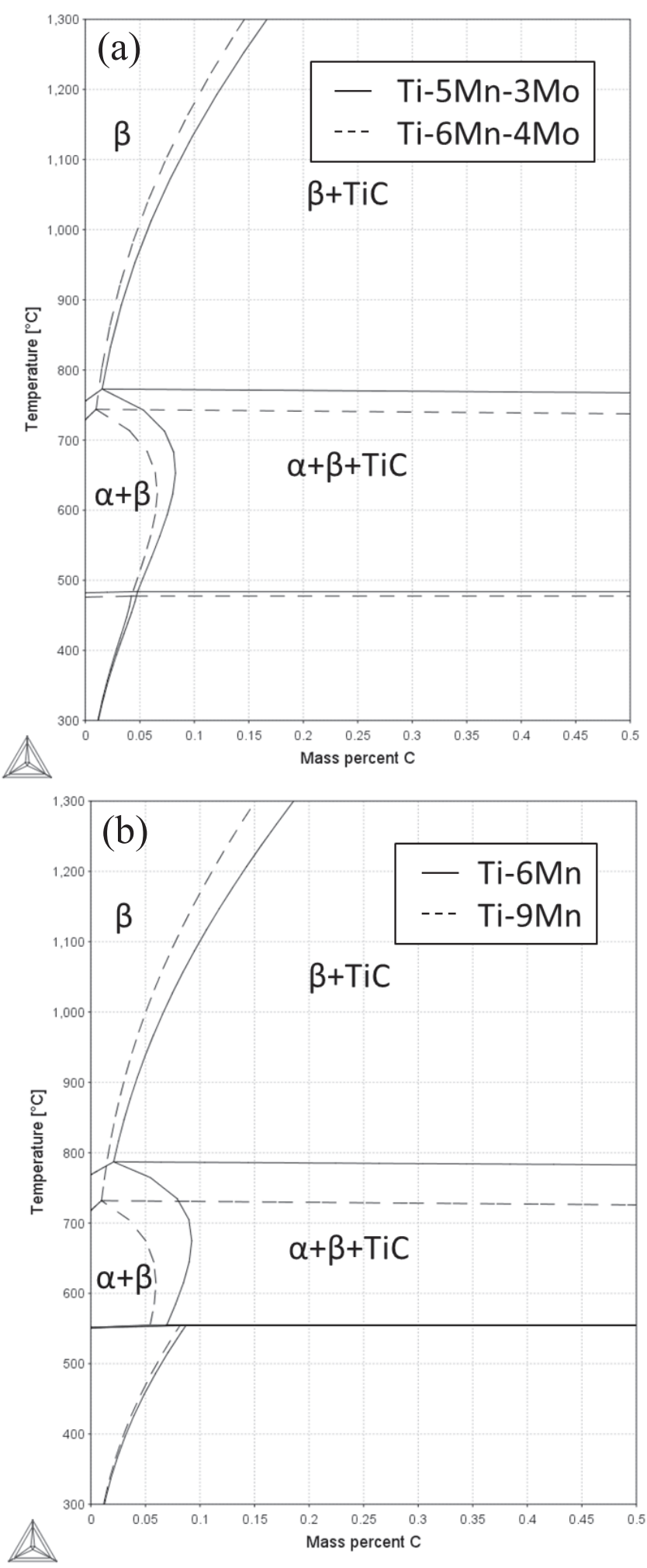

Fig. 14 Estimated solubility of C in (a) TMM-53 and TMM-64, and (b) Ti-6Mn and Ti-9Mn alloys.

thus decreasing the hardness measurements ${ }^{31,32)}$. Carbides can cause precipitation strengthening, which tends to increase the $H v^{31,32)}$. O concentrations, $V_{p o}$, and $V_{p r}$ are relatively constant among the TMM alloys, thus, no significant contribution from these factors can be observed among the TMM alloys fabricated by MIM. Compared to the TMM alloys fabricated by CCLM, the effects of solid solution hardening from 
the $\mathrm{O}$, the smaller amounts of $\omega$ phase, the presence of pores, and the precipitation hardening from the carbides balance each other so that the $H v$ of the alloys fabricated by MIM is comparable or lower than that of the alloys fabricated by CCLM.

The above-mentioned factors also affect the Young's modulus. The direct effects of $\mathrm{Mn}$ and Mo is negligible, because in the current concentrations they do not affect the electron-to-atom ratio of the alloys sufficiently in order to affect the Young's modulus ${ }^{38)}$. More predominantly, as the volume fraction of the athermal $\omega$ phase decreases, it should cause the $E$ to decrease. However, $\mathrm{O}$ can affect $E$ by affecting the interatomic distance ${ }^{39)}$, and also by inhibiting the formation of the $\omega$ phase $\mathrm{e}^{40)}$. Because of the inhibition of the formation of the $\omega$ phase (especially in TMM-53 and TMM-54, which would be expected to show a higher $E$ ), the TMM alloys fabricated by MIM have similar $E$ (84-88 GPa), with TMM-54 showing the lowest $E$ among them. Further, compared to the TMM alloys fabricated by CCLM ${ }^{23)}$, the amount of athermal $\omega$ phase is lower for all alloys fabricated by MIM, leading to lower Young's moduli. The presence of pores causes $E$ to decrease $^{41)}$. On the other hand, the presence of carbides, which have a higher Young's modulus, causes $E$ to increase. However, the $V_{p r}$ is believed to be too small to have a significant effect over the $E$ of the TMM alloys. Further, because $V_{p o}$, and $V_{p r}$ are relatively constant among the TMM alloys, it is believed that the influence of pores and carbides is relatively constant over $E$ among the TMM alloys fabricated by MIM.

The UTS of the TMM alloys fabricated by MIM shows the same trend as that observed for the hardness; thus, the predominant factors over the UTS are similar. The decreasing amount of athermal $\omega$ phase decreases the UTS, while the increasing $\mathrm{Mn}$ and Mo contents increase the solid solution strengthening effect and the UTS. Further, similar to the TiMn alloys fabricated by MIM, high O content and the presence of carbides can increase the UTS, while the presence of pores can decrease it. Overall, the low elongation of the TMM alloys is attributed to the high $\mathrm{O}$ content, and the presence of pores and carbides, which tend to decrease the ductility of the alloys ${ }^{31,40,41)}$. The increase in elongation from $2.0 \%$ in TMM53 to $5.0 \%$ in TMM-64 is attributed to the decrease in the amount of pores in the alloys, from $6.4 \%$ in TMM-53 to $4.8 \%$ in TMM-64.

Unlike the Ti-Mn alloys fabricated by MIM, every TMM alloy shows fracture surfaces predominantly covered by dimples (Fig. 9), which are associated with ductile fracturing, and were also observed in the TMM alloys fabricated by CCLM. Further, while carbide particles were present in some of the fracture surfaces (not shown here), their presence was not consistent. As the $V_{p r}$ is considerably low, around or lower than $0.6 \%$, it is difficult to determine if the carbides have any meaningful contribution to the fracture of the alloys. The presence of deformation-induced $\alpha$ " martensite in both TMM-53 and TMM-54 (Fig. 11), as well as deformation-induced $\{332\}<113>$ twins (Fig. 10 and Fig. 11) in TMM-54, indicates that TMM alloys fabricated by MIM have ductile deformation mechanisms. Thus, generally, the low elongation of the TMM alloys fabricated by MIM is mainly attributed to the high $\mathrm{O}$ content, and the presence of pores and carbides.

The almost constant difference between the $\sigma_{B}$ and $\sigma_{0.2}$ indicates there is work hardening upon tensile deformation in each alloy. For the cases of TMM-53, TMM-54 and their respective CCLM-fabricated counterparts ${ }^{23)}$, the difference is smaller because the amount of $\{332\}<113>$ twins, which help to improve the work hardening, is significantly reduced ${ }^{27,42)}$. Higher $\mathrm{O}$ contents have been shown to inhibit twin formation $^{40)}$. However, the larger amount of $\alpha$ " martensite, which also helps to improve work hardening ${ }^{42)}$, partially compensates the reduction in work hardening caused by the smaller amount of twins. On the other hand, TMM-63 and TMM-64 show larger differences between the $\sigma_{B}$ and $\sigma_{0.2}$ than those of their CCLM-fabricated counterparts ${ }^{23)}$. However, none of the MIM-fabricated or CCLM-fabricated TMM-63 or TMM-64 show twinning. In this case, the difference between the $\sigma_{B}$ and $\sigma_{0.2}$ is assumed to be caused by the relatively larger amount of deformation-induced $\omega$ phase which forms in the MIM-fabricated alloys. This is possible because, while high $\mathrm{O}$ contents can directly inhibit the formation of the deformation-induced $\omega$ phase, it can also inhibit the formation of the athermal $\omega$ phase, thereby indirectly leaving more possible nucleation sites for the deformation-induced $\omega$ phase $\mathrm{p}^{40)}$. Thus, the work hardening of TMM-53 is mostly influenced by the formation of deformation-induced $\alpha$ " martensite, while that of TMM-54 is also influenced by some amount of mechanical twinning. The work hardening of TMM-63 and TMM-64 is mostly influenced by the formation of deformation-induced $\omega$ phase.

Compared to the TMM alloys fabricated by $\mathrm{CCLM}^{23)}$, the TMM alloys fabricated by MIM show both lower Vicker hardness and Young's moduli, but higher tensile strength (except for TMM-63). Considering that $\mathrm{P} / \mathrm{M}$ products are expected to have lower strength compared to cast and wrought products $^{31,43)}$, the obtained results are favorable for biomedical applications. However, the elongation of the present TMM alloys is critically low, even when the deformation features are predominantly ductile.

Among the present TMM alloys, TMM-63 shows the best combination of mechanical properties. Its hardness, Young's modulus, $0.2 \%$ proof stress, and UTS are comparable or superior than those of most other Ti-Mn and TMM alloys developed for biomedical applications, and are superior to those of wrought Ti-64 ELI. However, like every alloy fabricated by MIM in this study, its ductility needs to be drastically improved. This can potentially be achieved by reducing the porosity and amount of carbides present, by using powders of higher purity, or by including processes such as hot isostatic pressing (HIP). However, these methods would result in increased alloy costs.

\section{Conclusions}

TMM alloys were fabricated by MIM (a low-cost P/M fabrication method), and their microstructures, mechanical properties, and deformation behaviors were investigated. Regarding the fabrication of Mo-added Ti-Mn system alloys by MIM,

(1) Mo addition promotes a significant reduction of the average grain diameters of the alloys, which are approximately half of the $D_{g r}$ of Ti-Mn alloys fabricated by MIM.

(2) OM and XRD analyses reveals the presence of only the 
$\beta$-phase for all the alloys, while the presence of an athermal $\omega$ phase in the alloys is confirmed by TEM observations. The amount of athermal $\omega$ phase decreases with increasing $M o_{e q}$.

(3) TMM-53 and TMM-54 show deformation-induced $\alpha$ " martensite after tensile deformation, while TMM-54 shows mechanical twinning after tensile deformation.

(4) Although the TMM alloys show predominantly ductile deformation mechanisms and fracture features, they also show low elongation. This is because the predominant factors over the elongation are the high $\mathrm{O}$ content, and the presence of pores and carbides, which are typically introduced by MIM fabrication of $\beta$-type Ti alloys.

(5) The Young's moduli and tensile strengths of TMM-53, TMM-54, and TMM-64 are more adequate for biomedical applications than those of their CCLM-fabricated counterparts, and those of wrought Ti-64 ELI. Their $\mathrm{Hv}$ values are also comparable to that of wrought Ti-64 ELI.

(6) TMM-63 $\left(\sigma_{B}=1143, \sigma_{0.2}=1077 \mathrm{MPa}\right.$, elongation = $3.9 \%$ ) shows the best combination of mechanical properties among the TMM alloys fabricated by MIM, with the greatest tensile strength. Its large UTS is attributed to the precipitation hardening effects of deformation-induced $\omega$ phase particles, and it possesses a hardness $(359 \mathrm{HV})$ comparable to that of TMM-63 fabricated by CCLM and a comparatively lower $E(86 \mathrm{GPa})$. Therefore, it shows attractive properties for biomedical applications as a lowcost alloy, but its elongation requires further improvement.

\section{Acknowledgments}

This study was supported in part by a Grant-in-Aid for Scientific Research (A) No. 24246111, a Grant-in-Aid for Young Scientists (B) No. 25820367 from the Japan Society for the Promotion of Science (JSPS), the Inter-University Cooperative Research Program "Innovation Research for Biosis-Abiosis Intelligent Interface" from the Ministry of Education, Culture, Sports, Science and Technology (MEXT), Japan, the Innovative Structural Materials Association (ISMA), Japan, and ICC-IMR of Tohoku University, Japan.

\section{REFERENCES}

1) M. Niinomi: Metall. Mater. Trans., A 33 (2002) 477-486.

2) M. Niinomi: J. Mech. Behav. Biomed. Mater. 1 (2008) 30-42.

3) M. Geetha, A.K. Singh, R. Asokamani and A.K. Gogia: Prog. Mater. Sci. 54 (2009) 397-425.

4) M. Niinomi: Mater. Sci. Eng. A 243 (1998) 231-236.

5) T. Kodama: Kokubyo Gakkai Zasshi 56 (1989) 263-288.

6) J.L. Domingo: Biol. Trace Elem. Res. 88 (2002) 97-112.

7) D.P. Perl: Environ. Health Perspect. 63 (1985) 149-153.

8) ATSDR (Agency for Toxic Substances and Disease Registry): Public Health Statement for Aluminum (2008) (online).

9) M. Niinomi, M. Nakai and J. Hieda: Acta Biomater. 8 (2012) 3888 3903.
10) R.M. Pilliar, H.U. Cameron, A.G. Binnington, J. Szivek and I. Macnab: J. Biomed. Mater. Res. 13 (1979) 799-810.

11) D.R. Sumner, T.M. Turner, R. Igloria, R.M. Urban and J.O. Galante: J. Biomech. 31 (1998) 909-917.

12) Y.L. Hao, S.J. Li, S.Y. Sun and R. Yang: Mater. Sci. Eng. A 441 (2006) $112-118$.

13) W. Guo, M.Z. Quadir, S. Moricca, T. Eddows and M. Ferry: Mater. Sci. Eng. A 575 (2013) 206-216.

14) D. Kuroda, M. Niinomi, M. Morinaga, Y. Kato and T. Yashiro: Mater. Sci. Eng. A 243 (1998) 244-249.

15) P.J. Bania: JOM 46 (1994) 16-19.

16) M. Niinomi, T. Hattori, K. Morikawa, T. Kasuga, A. Suzuki, H. Fukui and S. Niwa: Mater. Trans. 43 (2002) 2970-2977.

17) L. Erdmann and T.E. Graedel: Environ. Sci. Technol. 45 (2011) 76207630 .

18) U.S. Department of Energy: Critical Materials Strategy (2011) (online).

19) P.F. Santos, M. Niinomi, K. Cho, M. Nakai, H. Liu, N. Ohtsu, M. Hirano, M. Ikeda and T. Narushima: Acta Biomater. 26 (2015) 366-376.

20) K. Cho, M. Niinomi, M. Nakai, H. Liu, P.F. Santos, Y. Itoh, M. Ikeda, M. Abdel-Hady Gepreel and T. Narushima: J. Alloy. Compd. 664 (2016) 272-283.

21) P.F. Santos, M. Niinomi, H. Liu, K. Cho, M. Nakai, Y. Itoh, T. Narushima and M. Ikeda: J. Mech. Behav. Biomed. Mater. 59 (2016) 497-507.

22) T. Saito: Adv. Perform. Mater. 2 (1995) 121-144.

23) P.F. Santos, M. Niinomi, H. Liu, K. Cho, M. Nakai, A. Trenggono, S. Champagne, H. Hermawan and T. Narushima: Mater. Des. 110 (2016) 414-424.

24) Y. Itoh, H. Miura, T. Uematsu, T. Osada and K. Sato: J. Solid Mech. Mater. Eng. 3 (2009) 921-930.

25) R. Boyer, G. Welsch and E. W. Collings: Materials Properties Handbooks - Titanium Alloys (ASM International, 1993).

26) E. Bertrand, P. Castany, I. Péron and T. Gloriant: Scr. Mater. 64 (2011) 1110-1113.

27) F. Sun, J.Y. Zhang, M. Marteleur, T. Gloriant, P. Vermaut, D. Laillé, P. Castany, C. Curfs, P.J. Jacques and F. Prima: Acta Mater. 61 (2013) 6406-6417.

28) S.-J. L. Kang: Sintering - Densification, Grain Growth and Microstructure (Butterworth-Heinemann, 2004).

29) R. P. Elliott: Armed Services Technical Information Agency/AD 290 336 (Arlington Hall Station 1962).

30) D. F. Heaney: Handbook of Metal Injection Molding (Elsevier Science, 2012).

31) R. German: Materials (Basel) 6 (2013) 3641-3662.

32) P.G. Esteban, L. Bolzoni, E.M. Ruiz-Navas and E. Gordo: Powder Metall. 54 (2011) 242-252.

33) A. Devaraj, S. Nag, R. Srinivasan, R.E.A. Williams, S. Banerjee, R. Banerjee and H.L. Fraser: Acta Mater. 60 (2012) 596-609.

34) W.F. Ho: J. Med. Biol. Eng. 28 (2008) 47-51

35) N.E. Paton and J.C. Williams: Scr. Metall. 7 (1973) 647-649.

36) C. Hammond and J. Nutting: Prog. Met. Phys. 7 (1977) 65-163.

37) H. R. Ogden and R. I. Jaffee: Office of Assistant Secretary of Defense for Research and Development (1955).

38) G.P. Tiwari and R.V. Ramanujan: J. Mater. Sci. 36 (2001) 271-283.

39) F. Geng, M. Niinomi and M. Nakai: Mater. Sci. Eng. A 528 (2011) 5435-5445.

40) H. Liu, M. Niinomi, M. Nakai, K. Cho, K. Narita, M. Şen, H. Shiku and T. Matsue: Acta Biomater. 12 (2015) 352-361.

41) D. Zhao, K. Chang, T. Ebel, M. Qian, R. Willumeit, M. Yan and F. Pyczak: J. Mech. Behav. Biomed. Mater. 28 (2013) 171-182.

42) M. Marteleur, F. Sun, T. Gloriant, P. Vermaut, P.J. Jacques and F. Prima: Scr. Mater. 66 (2012) 749-752.

43) G.C. Obasi, O.M. Ferri, T. Ebel and R. Bormann: Mater. Sci. Eng. A 527 (2010) 3929-3935. 